\title{
ORIGINAL ARTICLE \\ Anti-psychotics adherence among out-patients with schizophrenia in Hong Kong
}

\author{
Christy LM Hui, ${ }^{1}$ Eric YH Chen, ${ }^{1}$ CS Kan, ${ }^{3} \mathrm{KC}$ Yip, ${ }^{4} \mathrm{CW}$ Law $^{2}$ and Cindy PY Chiu ${ }^{2}$ \\ ${ }^{1}$ University of Hong Kong, ${ }^{2}$ Queen Mary Hospital, ${ }^{3}$ Castle Peak Hospital, ${ }^{4}$ Kowloon Hospital, Hong Kong
}

(Received for publication on September 7, 2005)

(Revised for publication on December 12, 2005)

(Accepted for publication on January 26, 2006)

\begin{abstract}
Objective: Adherence to antipsychotic treatment is an important aspect of long term management of schizophrenia and other related psychotic disorders. The evaluation of adherence is often difficult in clinical settings. This study compared patients self-reports and clinician judgment of adherence behavior and explored their relationship with attitudes toward taking medication. Methods: Clinician rated questionnaire and matching patient self rated questionnaires were administered to 508 consecutive outpatients and their clinicians in four hospital clinic sites in Hong Kong. Items in the questionnaires address adherence behaviors as well as attitudes toward medication. In addition, clinicians also estimate the presence of factors unfavorable to adherence such as substance abuse, cognitive impairments etc. Results: Non-adherence was reported in at least $26 \%$ of patients. Clinician's detection of non-adherence behaviors achieved relative high specificity (0.84) but has low sensitivity (0.33). A logistic regression analysis on patients' decision to stop medication revealed that significant predictors include patients' reported lack of perception from the benefits of medication, patients' reported negative feelings associated with regular medication and younger age. Conclusion: Non-adherence is a widespread phenomenon affecting a significant proportion of outpatients under treatment. Clinicians have relatively low sensitivity in detecting which patients are non-adherent. Non-adherence behavior is predicted by level of awareness of ilness, perceived benefits of medication, negative feelings toward medication and age. (Keio J Med 55 (1): 9-14, March 2006)
\end{abstract}

Key words: medication adherence, compliance, schizophrenia

\section{Introduction}

Medication adherence is a significant challenge in the management of many chronic disorders. ${ }^{1}$ For patients with schizophrenia, although regular antipsychotic medication is essential for symptomatic treatment and relapse prevention, ${ }^{2-4}$ non-compliance rates for persons discharged from inpatient care are found to be at least $50 \%$ after 1 year and $75 \%$ at 2 years. ${ }^{5}$ The problems with adherence are not confined to patients with chronic illness, in first episode schizophrenia, 39\% of patients were estimated to be non-adherent and $20 \%$ were estimated to be inadequately adherent. ${ }^{4}$ It is generally accepted that the risk of relapse can be as much as five times higher upon discontinuation of treatment. ${ }^{6}$ Therefore, non-adherence to antipsychotic medications has important implications over the risk of exacerbation and relapse.

Agarwal et al. found that non-compliant patients were generally younger, had a shorter duration of illness and had an episodic course of illness. They also reported less side-effects, made more incorrect attributions to current positive symptoms and had a more negative subjective view of medication. ${ }^{7}$ Coldham et al. confirmed that non-adherent patients were younger, had an earlier age of onset and less likely to have a family member involved in treatment. In addition, nonadherent patients demonstrated more positive symptoms, more relapses, more alcohol and cannabis use, reduced insight, and poorer quality of life. ${ }^{4}$ Kampman et al. found that determinants of non-compliance included side effects, male gender, young age, lack of

Reprint requests to: Dr. Eric YH Chen, Department of Psychiatry, The University of Hong Kong, Queen Mary Hospital, Pokfulam Road, Hong Kong, e-mail: eyhchen@hkusua.hku.hk 
social activities, and high PANSS (Positive and Negative Syndrome Scale) total score but with a low score on PANSS positive symptoms. ${ }^{8}$ The most common patientreported barriers were related to the stigma of taking medications, side-effects, forgetfulness, and lack of social support. ${ }^{9}$

Effective intervention for non-adherence includes behavioral, educational and cognitive approaches. ${ }^{3,10-12}$ One of the difficulties related to intervention for non-adherence is the lack of information over the optimal screening method for patients at increased risk of non-adherence. In real life clinical practice, often the clinician's judgments are relied upon to identify patients with potential adherence problems. Little is known, however, about the sensitivity and the specificity of the clinicians screening evaluations.

In this study, we would like to compare clinicians' detection of non-adherence behavior against patients self report in a sample of outpatients with schizophrenia. In addition, we wish to explore the relationship between reported non-adherence behavior and factors such as gender, age, illness duration, and whether atypical or conventional medication is involved as well as non-adherence attitudes toward medication.

\section{Methods}

\section{Participants}

Patients were recruited from four outpatient clinics in Hong Kong. They were Queen Mary Hospital (QMH), Pamela Youde Nethersole Eastern Hospital (PYNEH), Castle Peak Hospital (CPH) and Kowloon Hospital (KH). Patients of QMH and PYNEH attended the Early Assessment Service for Young People with Psychotic Disorders (EASY), in which, patients were mainly younger and had shorter illness durations. Patients in the other clinics attended the general adult psychiatry clinics and were older and had longer illness durations. 508 out of $590(86 \%)$ patients approached for the study had participated. The recruitment period lasted for 2 months in total.

\section{Procedure and measures}

Patients must be over 18 and clinically diagnosed with schizophrenia, schizoaffective disorder or schizophreniform disorder. After providing written informed consent, patients were required to complete an abridged version of the Medication Adherence Rating Scale (MARS), ${ }^{13}$ in which, the six selected items that pertained to the adherence of patients to medication behaviors and attitudes (which went through satisfactory translation and back-translation procedures into Cantonese, Cantonese version available upon request).
At the same time, their corresponding clinicians would complete a questionnaire, and some of the items were comparable to the MARS i.e. matching adherence behavior items that enable further comparison. The 10 items included adherence behaviors, attitudes and factors that may affect medication adherence. Other relevant information that was collected included Clinical Global Impression (CGI), duration of illness and the class of medication used (i.e. either conventional or atypical).

\section{Data analysis}

Statistical analyses were carried out using the Statistical Package for Social Sciences (SPSS) version 12.0. First of all, results of clinicians and patients on respective medication adherence items were reported in frequency. Then, the relationship between the clinicians' evaluations and the patients' perceptions was assessed by using Chi-square tests. Significant items were then further examined by comparing their sensitivity and specificity levels. To assess the associations between factors (gender, age, illness duration and class of medication) and the reported level of adherence, patients were divided into groups to compare (using Chi-square tests) their effects on different adherence factors. A binary logistic regression was carried out to explore relative contribution of relevant factors toward patients' self-reported adherence behavior. Model chi-square and the Hosmer and Lemeshow Goodness-of-Fit Test were used to determine the extent to which the regression model's estimates fit the data. The Nagelkerke Rsquare was presented to indicate the overall strength of association. A classification table was calculated to summarize the proportion of cases that would be correctly categorized by the model.

\section{Results}

\section{Participants}

A total of 508 participants (256 male and 252 female) with a mean age was $37.8(\mathrm{SD}=15.2)$ years took part in the study. The mean duration of illness was 11.1 years $(\mathrm{SD}=10.5) .61 \%$ were taking conventional antipsychotic medication and 39\% were taking atypical antipsychotic medication.

\section{Descriptive results}

Patients' reported adherence behaviors and attitudes toward medication

There was $27 \%$ of patients claimed that they had forgotten to take the medication sometimes. $19 \%$ of 
patients reported that they had decided to stop taking the medication on their own accord. $13 \%$ of patients admitted to taking the medication only when they were feeling unwell. On the other hand, 39\% patients felt their situation deteriorated when they did not take their medication. A high percentage $(81 \%)$ of patients felt that medication was beneficial, however, about one third $(30 \%)$ of patients felt negative about the need to take medication regularly.

\section{Clinicians' evaluations of adherence}

Based on clinicians' evaluation, they believed that about $21 \%$ of patients had forgotten to take the medication as prescribed and had stopped taking the medication of their own accord. Clinicians also believed that $24 \%$ of their patients had irregular daily routines that might affect adherence. Clinicians' also considered that $40 \%$ of patients needed reminders from others in order to take medication as prescribed. In addition, about half $(46 \%)$ of the patients was rated by clinicians to be unaware of their illness. $21 \%$ of patients was judged to have cognitive deterioration which might affect their compliance. On the other hand, clinicians suspected only $5 \%$ of their patients to have abused alcohol or drugs. Besides, clinicians thought that $18 \%$ of their patients felt embarrassed or upset about having to take tablets regularly. Clinicians felt that about one third $(31 \%)$ of patients, when they felt better, would consider that medication was unnecessary and stopped taking them.

\section{Comparisons between patients' and clinicians' percep- tions}

Clinicians' and patients' ratings on behaviors were compared with regards to two measures of adherence behavior: (A) forgetting to take medication and (B) deciding to stop medication on their own. Patients' self report non-compliant behaviour were taken as a reference, providing a pragmatic proxy measure for true non-compliant behaviour which is difficult to measure objectively on a large scale.

\section{A. Forgetting to take medication}

Concerning whether patients had forgotten to take the medication, the contrast between patients' selfreport and clinicians' judgment is shown in Table 1.

The sensitivity rate is 0.33 which means that the clinicians were only able to identify one third of the patients who themselves admitted to forgetting to take their medication. The specificity rate was 0.84 , which implied that in $84 \%$ patients who did not report forgetting (i.e. remember) the medication, clinicians' did not feel there is a problem.
Table 1 The Relationship between Clinicians' and Patients' Perceptions on Forgetting to Take Medication

\begin{tabular}{lcrrr}
\hline \hline Forgetting to take medication & \multicolumn{2}{l}{$\begin{array}{l}\text { Patients' } \\
\text { self-reports }\end{array}$} & Total \\
\cline { 2 - 3 } & & No & Yes & \\
\hline Clinicians' judgments & No & 313 & 91 & 404 \\
\multirow{2}{*}{ Total } & Yes & 60 & 44 & 104 \\
& & 373 & 135 & 508 \\
\hline
\end{tabular}

Table 2 The Relationship between Clinicians' and Patients' Perceptions on Deciding to Stop Medication

\begin{tabular}{lllll}
\hline \hline Deciding to stop medication & \multicolumn{2}{l}{$\begin{array}{l}\text { Patients' } \\
\text { self-report }\end{array}$} & Total \\
\cline { 2 - 3 } & & No & Yes & \\
\hline Clinicians' judgments & No & 337 & 66 & 403 \\
& Yes & 74 & 31 & 105 \\
Total & & 411 & 97 & 508 \\
\hline
\end{tabular}

B. Deciding unilaterally to stop medication

Table 2 shows the comparison between patients self report and clinician judgment on whether there had been a unilateral decision to stop medication by patients.

The sensitivity of clinicians detecting patients who stopped medication on their own was 0.32 , which means that only $32 \%$ of patients who had stopped taking the medication on their own were identified by their clinicians. Besides, the specificity was 0.82 , that is, $82 \%$ of patients who did not stop the medication by themselves were considered as such by their clinicians.

\section{Factors associated with the reported level of adherence}

\section{Gender}

Gender effect could not be found between clinicians' and patients' ratings on factors affecting medication adherence. In other words, there was no significant different between male and female patients in their reports on adherence behaviors. However, variables affecting adherence in clinicians' items had significant results. More males $(30 \%)$ than females $(18 \%)$ were considered to have irregular routine that would affect daily adherence (Chi-square $=9.8, \mathrm{p}=0.002$ ). Moreover, clinicians' considered that there were more males $(8 \%)$ than females $(2 \%)$ had a problem with alcohol or drugs abuse $($ Chi-square $=10.93, \mathrm{p}<0.001)$.

\section{Age}

To examine the effects of age on adherence behaviors and attitudes, patients' were divided into five 


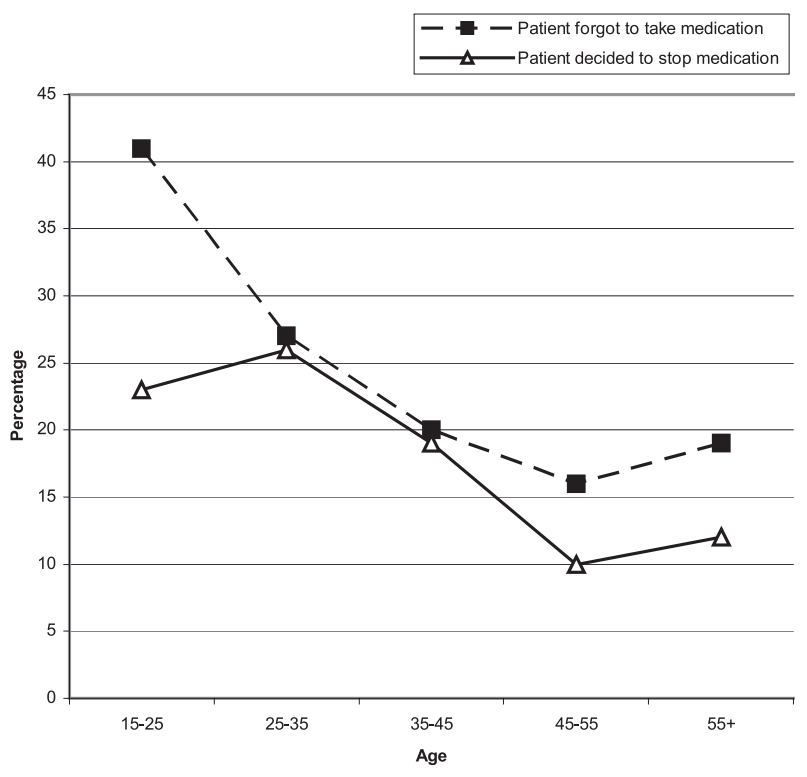

Fig. 1 Age effects on adherence behaviors.

groups according to their age. The first group was 15 to 25 years; the second group was 25 to 35 years; the third group was 35 to 45 years; the fourth group was 45 to 55 years and the last group was 55 years onwards. Figure 1 showed the items with significant age effects.

Patients report of having forgotten to take the medication were most prevalent among the youngest group $(41 \%)$, this trend decreased with increasing age (Chisquare $=24.1, \mathrm{p}<0.001)$. Similarly, decision to stop the medication on their own was more often found in younger patients (Chi-square $=11.74, \mathrm{p}=0.019)$.

In addition, patients generally tended to believe that medication is beneficial, with older patients showing a relatively higher rate of endorsement (Chi-square $=$ 14.9, $\mathrm{p}=0.005)$. Likewise, younger patients tended to feel more negative about taking regular medication (Chi-square $=13.95, \mathrm{p}=0.007)$.

\section{Illness Duration}

Patients were divided into two groups according to duration of illness using 5 years as the cutoff. Patients' illness duration of less than 5 years was more likely to stop the medication $(24 \%$ vs $15 \%$, Chi-square $=6.8$, $\mathrm{p}=0.012$ ). This pattern was observed with different cutoff points between 5 to 10 years. None of the other variables were related to illness duration.

\section{Type of medication}

The 508 patients were then divided into two groups (conventional or atypical medication). It appeared that patients taking the atypical medications were more likely to report stopping the medication as compared to the group taking only the conventional medication $(25 \%$ vs $15 \%$; Chi-square $=7.2 ; p=0.007)$. However, patients taking atypical medication were younger $(t=7.24, p<0.001)$ and had shorter illness durations $(\mathrm{t}=5.71, \mathrm{p}<0.001)$, and both of these factors were associated with increased tendency to stop medication. We attempted to remove this potential confound by dividing patients into five groups according to age and compared between patients taking atypical and conventional medication within each age group. We found no significant difference in the likelihood to stop medication between patients taking atypical and conventional medication. Likewise, patients were compared between two groups according to illness duration ( $<5$ years and $>5$ years). We again found no significant difference in the likelihood to stop medication between patients taking atypical and conventional medication within each group.

\section{Logistic regression results}

As the dependent variable (patients reported selfinitiated decision to stop medication) is dichotomous, a binary logistic regression analysis was carried out to investigate relative contribution of the independent variables. The independent variables were age, illness duration, attitude items from the clinician and the patient rated scales that did not involve behavior, and did not involved circularity i.e. items that were only ratable when there had been a decision to stop medication.

The regression model has a model chi-square of $40.68(\mathrm{p}<0.001)$. The Hosmer and Lemeshow Goodness of Fit Test has a Chi-Square of 7.1, $\mathrm{p}=0.525$. These suggested that the model's estimates fit the data to an acceptable level. The R-square (Nagelkerke) for the regression model was 0.124 . The model correctly classified $97.3 \%$ of patients who did not report stopping medication and $7.2 \%$ of patients who decided to stop medication (overall percentage 80.1\%). Patients' reported lack of medication benefit, and negative feelings toward taking regular medication, and younger age were significant predictors of patients' report of selfinitiated decision to stop medication, with odds ratios of $0.46,2.9$, and 0.98 respectively (Table 3 ).

\section{Discussion}

In this study, we compared patients' self-report with clinician's judgment of non-adherence. We found that clinicians are relatively accurate in judging patients without non-adherence problems but are less effective in identifying patients with non-adherence problems. Besides, the overall rate of non-adherence was around $20 \%$ in both clinicians' estimation and patients' reports. This indicates that the problem may not be simply one of under-recognition by the clinicians. Instead, the difficulty lies in the identification of the individuals with non-adherence problems. 
Table 3 Logistic Regression Statistics for Prediction of Clinicians and Patients' Attitudes Toward Medication Adherence to Patients' Decision to Stop Taking the Medication $(\mathrm{n}=508$, binary logistic regression analysis, stepwise forward)

\begin{tabular}{|c|c|c|c|c|c|c|c|c|}
\hline & \multirow[t]{2}{*}{ B } & \multirow[t]{2}{*}{ S.E. } & \multirow[t]{2}{*}{ Wald } & \multirow[t]{2}{*}{$\mathrm{df}$} & \multirow[t]{2}{*}{ Sig. } & \multirow[t]{2}{*}{$\operatorname{Exp}(B)$} & \multicolumn{2}{|c|}{$95.0 \%$ C.I. for $\operatorname{EXP}(B)$} \\
\hline & & & & & & & Lower & Upper \\
\hline $\begin{array}{l}\text { Patients perceived } \\
\text { benefit of } \\
\text { medication }\end{array}$ & -.777 & .265 & 8.607 & 1 & .003 & .460 & .274 & .773 \\
\hline $\begin{array}{l}\text { Patient negative } \\
\text { feelings toward } \\
\text { regular medication }\end{array}$ & 1.064 & .240 & 19.621 & 1 & .000 & 2.898 & 1.810 & 4.641 \\
\hline Age & -.016 & .008 & 4.074 & 1 & .044 & .984 & .968 & 1.000 \\
\hline Constant & -.661 & .385 & 2.956 & 1 & .086 & .516 & & \\
\hline
\end{tabular}

It is worth noting that clinicians' judgment in this study closely resembles real life clinical situations, in which, in both cases clinicians have access to medical notes and information provided by patients and informants during interviews. Despite the ability to access information from a number of sources, clinicians' evaluation of patients' non-adherence is still suboptimal, as revealed in the discrepancies between clinicians' judgments and patients' self-reports of non-adherence. Therefore, our results show that it is not adequate to rely solely on clinicians' judgments in identifying patients with potential adherence problems. The need for more refined screening assessment is in line with the previous suggestions that screening should consist of assessment of a wider range of factors such as knowledge and beliefs about the illness, as well as service utilization patterns.

In this study we compared patients' self-report with clinician judgment. It is recognized that patients' selfreport may still represent an under-reporting of the magnitude of the problem. Though compared to interview, self-report questionnaires are less prone to suppression of undesirable responses. Therefore it offers a better approximation to the true adherence status compared with open interview.

We found that out-patients in Hong Kong who report non-adherence behaviors have a specific profile. We found that non-adherent patients are likely to be younger in age. This finding is consistent with results from a number of previous studies. ${ }^{5,15,16}$ In a naturalistic sample, younger age is likely to be associated with a shorter illness duration. Indeed we also find that patients with a shorter illness duration tended to have more non-compliance behaviors, as was also observed in previous study. ${ }^{16}$ Two mechanisms may underlie this observation. The first mechanism may involve patients early in the course of their illness being more willing to take risk to find out if they could remain well without medication, especially before they encounter repeated episodes of relapses. The second mechanism relates to selection bias of an outpatient population, in which patients with longer illness duration and is still regular in clinic attendance are more likely also to be relative compliant with their treatment.

Patients' negative attitude toward regular medication is found to be a significant factor associated with reported non-adherence behavior. This replicates a finding that has been reported in several previous studies. ${ }^{16^{-18}}$ Likewise, the perceived benefit of medication is also related to adherence behavior, consistent with previous findings. ${ }^{15-17,19}$

Unlike a number of other studies in Western settings, ${ }^{8,15}$ we do not find that male patients report more non-adherence behaviors. Flaskerud and $\mathrm{Hu}$ found that substance abuse was lower for Asians than for white, black, and Latino groups. ${ }^{23}$ The overall rate of substance abuse is also estimated to be low in our sample. The relationship between substance abuse and noncompliance may contribute to the observed excess of non-adherence in male patients in other sites. The low prevalence of substance abuse may partially explain the lack of gender effect in our sample.

We found that patients on atypical antipsychotic medication reported more non-adherent behaviors. This is contrary to our initial expectation, as atypical anti-psychotics are generally considered to have a more favorable adverse effect profile ${ }^{24}$ and are associated with better adherence behavior. ${ }^{25,26}$ However, this relationship has been challenged by some studies. ${ }^{27} \mathrm{~A}$ more important reason for the discrepancy may lies with the selective prescribing practice in which younger patients with shorter illness duration are more likely to be prescribed atypical anti-psychotics medication. Since both youth and short illness duration are associated with increased noncompliance, these factors may mediate the observed trend for patients on atypical antipsychotics to appear more non compliant. This possibility is confirmed by our further analysis comparing patients on atypical and convention anti-psychotics within groups with similar age and illness duration. 
While this study provides unique information allowing for an assessment of clinical judgment of nonadherence compared to patients' self-reports, there are several limitations. Firstly, patients' self-reports are not the most accurate measure for medication adherence. Despite the advantages of using a self-administered questionnaire, under-reporting may still occur. Therefore, the present $20 \%$ non-adherence rate represents the minimum level. In this study, informant information is taken up by the clinician and contributed to the clinician's judgment. Informant perspective might be more richly captured by a separated questionnaire. While the sample size is substantial in this study, the range of information obtained for each individual is selective. This enables us to identify certain relationship of interests, but not other potentially relevant factors such as social support and ethnicity.

This cross-section study of a large sample of outpatients in Hong Kong enabled the evaluation of sensitivity and specificity of clinical screening for nonadherence behavior. The result suggests that clinical screening on its own is inadequate in identifying patients at risk of non-adherence problems. More inclusive screening instruments are required to enhance detection of non-adherence. Assessment of patients' attitudes to medication, perceived benefits of medication and insight into the illness may be included as items closely related to adherence behavior. Screening should be particularly vigilant for younger patients with short illness duration. Good clinician-patient relationship, as well as specific intervention models may facilitate the detection and modification of non-adherence in patients.

Acknowledgement: We acknowledge partial support for this study from an unrestricted education grant from Janssen Pharmaceuticals.

\section{References}

1. Sabaté E: Adherence to long-term therapies: evidence for action. Geneva: World Health Organization; 2003

2. Fenton WS, Blyler CR, Heinssen RK: Determinants of medication compliance in schizophrenia: empirical and clinical findings. Schizophr Bull 1997; 23: 637-651

3. Dolder CR, Lacro JP, Leckband S, Jeste DV: Interventions to improve antipsychotic medication adherence: review of recent literature. J Clin Psychopharmacol 2003; 23: 389-399

4. Coldham EL, Addington J, Addington D: Medication adherence of individuals with a first episode of psychosis. Acta Psychiatr Scand 2002; 106: 286-290

5. Bebbington PE: The content and context of compliance. Int Clin Psychopharmacol 1995; 9 Suppl 5: 41-50

6. Dixon LB, Lehman AF: Family interventions for schizophrenia. Schizophr Bull 1995; 21: 631-643

7. Agarwal MR, Sharma VK, Kishore Kumar KV, Lowe D: Noncompliance with treatment in patients suffering from schizophrenia: a study to evaluate possible contributing factors. Int $\mathbf{J}$
Soc Psychiatry 1998; 44: 92-106

8. Kampman O, Laippala P, Vaananen J, Koivisto E, Kiviniemi P, Kilkku N, Lehtinen K: Indicators of medication compliance in first-episode psychosis. Psychiatry Res 2002; 110: 39-48

9. Hudson TJ, Owen RR, Thrush CR, Han X, Pyne JM, Thapa P, Sullivan G: A pilot study of barriers to medication adherence in schizophrenia. J Clin Psychiatry 2004; 65: 211-216

10. Knapp M: Schizophrenia costs and treatment cost-effectiveness. Acta Psychiatr Scand Suppl 2000; 407: 15-18

11. O'Donnell C, Donohoe G, Sharkey L, Owens N, Migone M, Harries R, Kinsella A, Larkin C, O'Callaghan E: Compliance therapy: a randomised controlled trial in schizophrenia. BMJ 2003; 327: 834

12. Kemp R, Kirov G, Everitt B, Hayward P, David A: Randomised controlled trial of compliance therapy. 18-month follow-up. Br J Psychiatry 1998; 172: 413-419

13. Thompson K, Kulkarni J, Sergejew AA: Reliability and validity of a new Medication Adherence Rating Scale (MARS) for the psychoses. Schizophr Res 2000; 42: 241-247

14. Nageotte C, Sullivan G, Duan N, Camp PL: Medication compliance among the seriously mentally ill in a public mental health system. Soc Psychiatry Psychiatr Epidemiol 1997; 32: 49-56

15. Fleischhacker WW, Oehl MA, Hummer M: Factors influencing compliance in schizophrenia patients. J Clin Psychiatry 2003; 64 Suppl 16: 10-13

16. Lacro JP, Dunn LB, Dolder CR, Leckband SG, Jeste DV: Prevalence of and risk factors for medication nonadherence in patients with schizophrenia: a comprehensive review of recent literature. J Clin Psychiatry 2002; 63: 892-909

17. Buchanan A: A two-year prospective study of treatment compliance in patients with schizophrenia. Psychol Med 1992; 22: 787-797

18. Donohoe G, Owens N, O’Donnell C, Burke T, Moore L, Tobin A, O'Callaghan E: Predictors of compliance with neuroleptic medication among inpatients with schizophrenia: a discriminant function analysis. Eur Psychiatry 2001; 16: 293-298

19. Ruscher SM, de Wit R, Mazmanian D: Psychiatric patients' attitudes about medication and factors affecting noncompliance. Psychiatr Serv 1997; 48: 82-85

20. Novak-Grubic V, Tavcar R: Predictors of noncompliance in males with first-episode schizophrenia, schizophreniform and schizoaffective disorder. Eur Psychiatry 2002; 17: 148-154

21. Budd RJ, Hughes IC, Smith JA: Health beliefs and compliance with antipsychotic medication. Br J Clin Psychol 1996; 35: 393 397

22. Garavan J, Browne S, Gervin M, Lane A, Larkin C, O'Callaghan E: Compliance with neuroleptic medication in outpatients with schizophrenia; relationship to subjective response to neuroleptics; attitudes to medication and insight. Compr Psychiatry 1998; 39: 215-219

23. Flaskerud JH, Hu LT: Relationship of ethnicity to psychiatric diagnosis. J Nerv Ment Dis 1992; 180: 296-303

24. Lindstrom E, Bingefors K: Patient compliance with drug therapy in schizophrenia. Economic and clinical issues. Pharmacoeconomics 2000; 18: 106-124

25. Diaz E, Neuse E, Sullivan MC, Pearsall HR, Woods SW: Adherence to conventional and atypical antipsychotics after hospital discharge. J Clin Psychiatry 2004; 65: 354-360

26. Menzin J, Boulanger L, Friedman M, Mackell J, Lloyd JR: Treatment adherence associated with conventional and atypical antipsychotics in a large state Medicaid program. Psychiatr Serv 2003; 54: 719-723

27. Loffler W, Kilian R, Toumi M, Angermeyer MC: Schizophrenic patients' subjective reasons for compliance and noncompliance with neuroleptic treatment. Pharmacopsychiatry 2003; 36: 105112 